\title{
Plummer-Vinson Syndrome with Improvement After Iron Repletion
}

Valerian Mwenda ${ }^{1}$, Jackson Njuguna ${ }^{2}$, Ancent Nzioka ${ }^{2}$.

1. Department of Internal Medicine and Endoscopy, Consolata Hospital Nkubu

2. Department of Pathology, School of Medicine, Kenyatta University.

Correspondence to: Dr. Valerian Mwenda P.0 Box 205-60200 Meru, Kenya. Email: valmwenda@gmail.com

\begin{abstract}
Plummer-Vinson Syndrome (PVS) is a rare disorder characterized by upper esophageal webs and iron deficiency anemia and is even more uncommon among people of African descent. We report a case of this syndrome in a middle-aged black woman, which improved on iron-repletion therapy alone. The patient presented with long-standing dysphagia and symptomatic anemia. Gastrointestinal endoscopy revealed an upper esophageal web arising from the anterior wall. She was started on iron therapy daily
\end{abstract}

\section{Introduction}

Plummer-Vinson syndrome (synonyms: PatersonKelly Syndrome, Paterson-Brown Kelly syndrome, Sideropenic dysphagia), is defined by the classic triad of dysphagia, iron deficiency anemia and esophageal webs (1). During the first half of the 20th century the syndrome was common among middle-aged Caucasian women (2), but the prevalence has fallen over the years. The fall in prevalence has largely been attributed to improvement in nutrition in areas where it used to be common, although in Africa where prevalence of iron deficiency and malnutrition is still high, the syndrome is rare (1). The syndrome has also been described in children and adolescents $(3,4,5)$. We present a case of a middle aged woman of African descent with classic symptoms of the syndrome and who showed a very remarkable improvement in the dysphagia after two months of iron repletion therapy. The case report is presented to remind clinicians of the existence of this rare disorder and the diagnostic approach, especially differentiating it with other causes of dysphagia.

\section{Case Presentation}

A 34 year old woman was referred to our health and after 2 months both the dysphagia and anemia had improved significantly. Therefore, though this is a relatively rare condition in Africa, it still warrants being part of the differential diagnosis for dysphagia especially in the setting of anemia.

Keywords: Plummer-Vinson Syndrome, Anemia, Dysphagia, Endoscopy

Ann Afr Surg. 2016;13(2): 81-3.

DOI: http://dx.doi.org/10.4314/aas.v13i2.11 facility with history of dysphagia to solids over the preceding three years and easy fatigability and intermittent dizziness over the preceding seven years. She could swallow liquids and very thin semi-solids, and reported gradual loss in weight. There was no cough or odynophagia. Her menses were regular and normal in amount. There was no history of melena stool or hematochezia. Her last delivery was 10 years prior to presentation. There was no familial history of similar symptoms or cancer. She had received short course iron supplementation in other hospitals with intermittent relief of the symptoms. In the referring hospital, upper intestinal endoscopy had been attempted but failed to successfully intubate the upper esophagus. On examination the patient was not in distress, had conjuctival pallor and tachycardia, and was moderately wasted. A full blood count showed a red cell count of $4.33 \times 10^{12} / \mathrm{L}($ reference; $4.10-5.90 \times 10^{12} / \mathrm{L}$ ), hemoglobin level of $6.5 \mathrm{~g} / \mathrm{dl}$, hematocrit of $23.5 \%$ (reference range 38$46 \%$ ), mean corpuscular volume of $59 \mathrm{fl}$ ( reference range, 80-100 fl) and mean corpuscular hemoglobin of $17.9 \mathrm{pg}$ ( reference range; $28-32 \mathrm{pg}$ ).The platelets, white cell count and differential counts were all within the reference ranges, as were the liver and renal 
function tests. While adequately anaesthetized, upper gastrointestinal endoscopy was done. A web was visualized on the upper esophagus, approximately 2 $\mathrm{cm}$ below the upper esophageal sphincter; it had a smooth appearance and a narrowed, eccentric lumen was visible. The endoscope was maneuvered slowly across the narrowed lumen, in the process tearing part of the web. The web was originating from the anterior wall of the esophagus as shown in figure 1 . The rest of the esophagus was normal. The stomach mucosa and wall were very transparent, with the mucosal blood vessels vey prominent, as depicted in figure 2 . The first and second parts of the duodenum were normal. Samples were obtained from the web and also from the stomach for histology. The photomicrograph of the histological appearance of tissue from the esophageal web is shown in figure 3 . The patient was managed with iron repletion therapy, with a target of $400 \mathrm{mg}$ of elemental iron daily and was seen after 2 months when she reported that she could now comfortably swallow solids, the easy fatigability had decreased and the hemoglobin level had improved to $11.6 \mathrm{~g} / \mathrm{dl}$. She is on follow-up and is to continue on haematinics for at least six months to build up iron stores. Written informed consent was obtained from the patient for publication of this case report and the accompanying images.

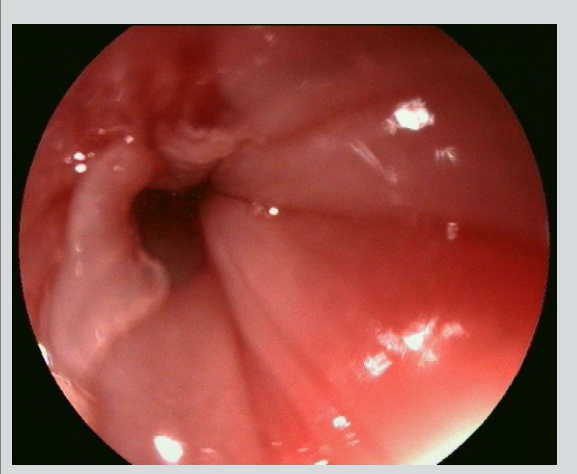

Figure 1: Endoscopic Image Showing the Web Arising From the Anterior Wall of the Proximal Esophagus.

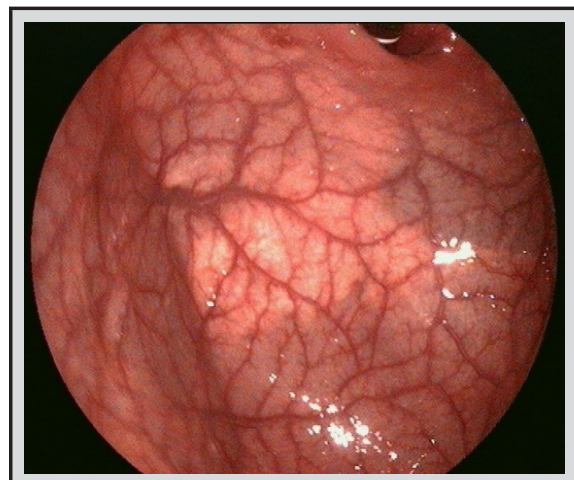

Figure 2: The Transparent Appearance of the Wall of the Proximal Stomach With Mucosal Vessels Clearly Visible.

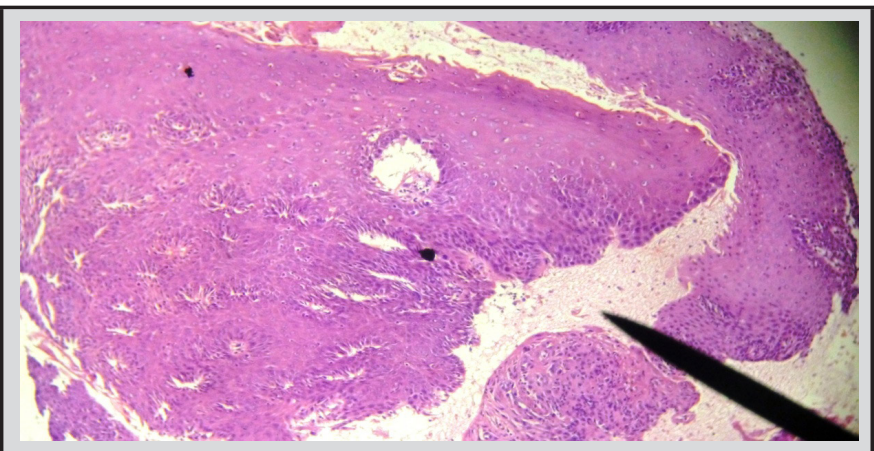

Figure 3: Photomicrograph of Esophageal Biopsy. Routine Hematoxylin and Eosin Stains X40 Magnification. Shows Atrophic Esophageal Mucosa with Alternating Areas of Hyperparakeratosis

\section{Discussion}

This case shows that Plummer-Vinson syndrome (PVS) still occurs in African settings, with typical demographic and clinical features. It usually presents with post-cricoid dysphagia, upper esophageal webs and iron deficiency anemia. The dysphagia in these patients is usually painless, intermittent or slowly progressive over years, limited to solids only and can sometimes be associated with weight loss as was the case in this patient (1). Other symptoms include those of iron deficiency and anemia such as koilonychia, angular cheilitis, easy fatigability and dizziness. This patient had reported symptoms of anemia for approximately seven years and dysphagia for the preceding three years. Other common causes of iron deficiency in middle-aged women in Africa were ruled out. The syndrome is more common in middle aged women, with a mean age at presentation of 47 years in an analysis of 28 English language case reports in published in the literature from 1998-2005 (1). The esophageal web in this patient was arising from the anterior wall of the proximal esophagus, causing incomplete obstruction of the lumen. Many conditions have been reported to be associated with the syndrome including rheumatoid arthritis, thyroid disease, celiac disease, genetic predisposition and malnutrition (1). The most probable mechanism of disease is thought to be iron deficiency because of three reasons: first, this finding is part of the classic triad; second, in most cases the dysphagia improves with iron supplementation and third, the alimentary tract is highly susceptible to iron deficiency by losing iron-dependent enzymes which in turn is speculated to cause mucosal degeneration and web formation (1). Most cases of PVS can be managed with iron supplementation alone (6), though some patients may require dilatation of the webs especially in significant esophageal obstructions or patients whose dysphagia persist despite iron repletion. Dilatation can be performed by either wire-guided dilators or balloon 
dilation. The syndrome has a favorable prognosis after either form of management, but since PVS has been noted to be a risk factor for squamous cell carcinoma of the esophagus or pharynx, long-term, close follow-up with surveillance upper gastrointestinal endoscopy is recommended $(7,8)$. Recent reports in African settings also show female preponderance and good response to conservative treatment $(9,10)$.

\section{Conclusion}

Plummer-Vinson syndrome, though a rare condition in Africa, still occurs and warrants being part of the differential diagnosis of dysphagia, especially in middle aged women. Since most patients will respond to iron repletion therapy, this conservative approach is recommended and dilatation performed only for resistant cases. Follow-up for patients with this condition is also recommended due to association with future development of cancer.

\section{Authors' contributions}

VM collected the patient's data, performed the endoscopy and prepared the initial manuscript. AN prepared, read and reported the histological slides. JN edited the final manuscript. All authors read and approved the final manuscript.

\section{Acknowledgements}

We highly acknowledge Mrs. Mary for retrieving and availing the patient's admission records and the patient for allowing us to present the case.

\section{References}

1. Novacek G. Plummer-Vinson Syndrome. Orphanet J Rare Dis. 2006; 1:36

2. Wynder EL, Hultberg S, Jacobsson F, et al. Environmental Factors in Cancer of the Upper Alimentary Tract: A Swedish Study with Special Reference to Plummer-Vinson (Paterson-Kelly) syndrome. Cancer. 1957;10 (3): 470-82.

3. Mansell N, Jani P, Bailey C. Plummer-Vinson syndrome-a rare presentation in a child. J Laryngol Otol. 1999;113(5): 475-6.

4. Anthony R, Sood S, Strachan D, et al. A Case of Plummer-Vinson Syndrome in Childhood. J Pediatr Surg 1999, 34(10): 1570-72.

5. Lopez RMJ, Robledo AP, Amarilla JA, et al. Sideropenic Dysphagia in an Adolescent. J Pediatr Gastroenterol Nutr. 2002;34(1): 87-90.

6. Hoffmann R, Jaffe P. Plummer-Vinson Syndrome: A Case Report and Literature Review. Arch Intern Med. 1995; 155(18): 2008-2011.

7. Chisholm M. The Association Between Webs, Iron and Post-Cricoid Carcinoma. Postgrad Med J 1974; 50(582): 215-19.

8. Messmann H. Squamous Cell Cancer of the Esophagus. Best Pract Res Clin Gastroenterol. 2001;15(2): 249-65.

9. Hefaiedh R, Boutreaa Y, Ouakaa-Kchaou A, et al. Plummer-Vinson Syndrome. Tunis Med. 2010; 88(10): 721-4

10. Adama B, Madoky M, Papa S, et al. Unusual Presentation of Plummer-Vinson Syndrome in Black African: A Report of Two Cases. Pan Afr Med J. 2014; 19: 145. 